\title{
Prediction of conversion to psychosis: review and future directions
}

\section{Previsão de conversão para psicose: revisão e perspectivas futuras}

\author{
Dylan G. Gee, Tyrone D. Cannon
}

Departments of Psychology and Psychiatry \& Biobehavioral Sciences - University of California, Los Angeles

\begin{abstract}
This article reviews recent findings on predictors of conversion to psychosis among youth deemed at ultra high risk (UHR) based on the presence of subpsychotic-intensity symptoms or genetic risk for psychosis and a recent decline in functioning. Although transition rates differ between studies, the most well powered studies have observed rates of conversion to full psychosis in the 30-40\% range over 2-3 years of follow-up. Across studies, severity of subthreshold positive symptoms, poorer social functioning, and genetic risk for schizophrenia appear to be consistent predictors of conversion to psychosis, with algorithms combining these indicators achieving positive predictive power $\geq 80 \%$. Nevertheless, a substantial fraction of UHR cases do not convert to psychosis. Recent work indicates that UHR cases who present with lower levels of negative symptoms and higher levels of social functioning are more likely to recover symptomatically and no longer meet criteria for an at-risk mental state. In general, it appears that about $1 / 3$ of UHR cases convert to psychosis, about $1 / 3$ do not convert but remain symptomatic and functionally impaired, and about $1 / 3$ recover symptomatically and functionally. Continued efforts to detect early risk for psychosis are critical for informing early intervention and provide increasing promise of delaying or even preventing the onset of psychosis.
\end{abstract}

Descriptors: Psychosis; Symptoms; Schizophrenia; Gene conversion; Algorithms

\section{Resumo}

$O$ presente artigo revisa os achados recentes sobre os preditores de conversão para psicose entre jovens considerados de ultra alto risco (UAR) com base na presença de sintomas de intensidade sub-psicótica e risco genético para psicose e declínio recente no funcionamento mental. Apesar das taxas de transiçāo serem diferentes entre os estudos, os estudos de mais peso encontraram taxas de conversáo para psicose entre $30 \%$ e $40 \%$ em pacientes acompanhados por dois a três anos. Entre os estudos, gravidade de sintomas positivos sub-clínicos, pior relacionamento social e risco genético para esquizofrenia parecem ser preditores consistentes de conversão para psicose, com algoritmos combinando esses indicadores alcançando poder preditivo positivo $\geq 80 \%$. Ainda assim, uma fração substancial de casos em UAR não converte para psicose. Trabalhos recentes indicam que casos em UAR que apresentam niveis mais baixos de sintomas negativos e niveis mais altos de bom relacionamento social apresentam maior probabilidade de recuperação dos sintomas e de não mais preencher os critérios para estado mental de risco. Em geral, parece que 1/3 dos casos de UAR convertem para psicose, cerca de $1 / 3$ não convertem, mas se mantém sintomáticos e com comprometimento funcional, e cerca de 1/3 apresentam recuperação sintomática e funcional. Esforços contínuos para detectar risco precoce para psicose sáo críticos para a intervenção precoce e para fornecer uma promessa cada vez maior de retardar ou até prevenir o inicio da psicose.

Descritores: Psicoses; Sintomas; Esquizofrenia; Conversão gênica; Algoritmos

\author{
Correspondence \\ Tyrone D. Cannon \\ UCLA - Psychology \\ 1285 Franz Hall Los Angeles California 90095 \\ United States \\ Tel: $(+001) 310-206-8765$ \\ Fax: (+001) 310-794-9740 \\ E-mail: cannon@psych.ucla.edu
}




\section{Introduction}

In the past two decades there has been increasing interest in the early detection of those at risk for psychosis, with the aims of improving understanding of mechanisms underlying risk progression and disease onset and allowing for early intervention. Consistent with this approach, indicated prevention that is targeted toward individuals expressing subthreshold symptoms represents a promising strategy for delaying or preventing psychosis and thus thwarting the debilitating effects of psychosis. Central to this effort is the development of systematic, empirically validated criteria for identifying individuals at elevated risk for psychosis. Through ongoing testing and refinement, research has led to the ultra high risk (UHR) approach, which has been shown to identify individuals at imminent risk for psychosis, thus allowing for enhanced prediction of outcomes and an empirical basis on which to inform early intervention strategies. However, substantial variability between studies and heterogeneity among UHR individuals have limited efforts to date. In addition, the majority of individuals identified as UHR do not convert to psychosis within the study periods, suggesting that the UHR criteria warrant continued refinement. These findings also lead to questions about the outcomes of non-converting individuals and whether these individuals require monitoring or early intervention. Here we review findings concerning prediction of psychoses in UHR samples and describe recent findings on the outcomes of non-converting individuals.

\section{Description of ultra high risk criteria and summary of conversion rates}

The UHR approach aims to prospectively identify individuals at imminent risk for the onset of psychosis by focusing on three primary categories of risk ${ }^{1}$. First, attenuated positive symptoms (APS) emphasize the onset or worsening of subthreshold positive symptoms within the past 12 months. Such symptoms are categorized into unusual thought content, suspicion/paranoia, perceptual abnormalities, grandiosity, and disorganized communication. The severity of each symptom type is rated based on its frequency, duration, impact on functioning, and extent of loss of insight. Brief intermittent psychotic symptoms (BIPS) constitute a second category of UHR criteria, which refers to the onset of transient suprathreshold psychotic symptoms within the past three months. That is, individuals meeting BIPS criteria experience positive symptoms that are of a psychotic intensity level but do not meet criteria for a DSM-IV Axis I psychotic disorder diagnosis. Genetic risk and deterioration (GRD) forms a third category of UHR syndrome, which is identified by genetic risk for psychosis and recent functional decline. GRD criteria have previously been operationalized as having a firstdegree relative with a psychotic disorder, or as having a diagnosis of schizotypal personality disorder, in addition to a decline in functioning within the past 12 months as measured by the Global Assessment of Functioning (GAF) score ${ }^{2}$. Two primary measures, the Comprehensive Assessment of At-Risk Mental
States (CAARMS ${ }^{3}$ ) and the Structured Interview for Prodromal Syndromes (SIPS ${ }^{4}$ ) have been validated to assess for UHR criteria, with slight differences in definitions.

Another approach to the early detection of risk for psychosis has been the use of basic symptoms, as measured by scales such as the Bonn Scale for the Assessment of Basic Symptoms (BSABS5) or Schizophrenia Prediction Instrument - Adult version (SPI-A ${ }^{6}$ ). Basic symptoms are defined as subjective, subtle disturbances that are experienced across various domains of mental functioning such as cognition, initiative, perception, and energy level ${ }^{7,8}$. These symptoms are not encompassed by positive symptom assessments. It has been hypothesized that basic symptoms might represent an early indication of a prodromal stage, whereas UHR criteria might signify a later prodromal stage ${ }^{8}$.

Studies employing criteria for an at-risk state have observed varying rates of transition to psychosis, ranging between 9-76\% depending on various factors. Table 1 provides a summary of transition rates observed among some of the best-powered studies to date. Extant research on transition to psychosis varies on a number of factors including study methodology, risk ascertainment criteria, sample characteristics, and frequency and duration of follow-up. For example, sample sizes have ranged from 13 to 291, and follow-up periods have lasted between 1 to 9 years (as reviewed by Olsen $\&$ Rosenbaum ${ }^{9}$ ). Some studies have noted a decreasing transition rate over the years ${ }^{8,10,11}$; however, the reasons for a possible decrease remain unclear. It may be that, in some locations, increased community outreach and educational efforts have led to ascertainment of individuals at an earlier stage of risk. Moreover, early intervention or case management provided at many clinical research centers may be facilitating symptom reduction. It is also important to note that, in relatively young cohorts, many participants have not yet reached the average age of psychosis onset ${ }^{11,12}$.

Several additional issues arise among efforts to identify individuals at increased risk for psychosis. In reviewing the extant literature on prediction of psychosis, the extent to which differing transition rates result from differences in selection criteria between studies remains unclear. For example, some studies differ on the consideration of negative symptoms, exclusion of the BIPS category of risk, and criteria for recency of onset or duration of positive symptoms. In addition, heterogeneity among at-risk individuals may hinder efforts to accurately predict the onset of psychosis. Variability in age at study entry also raises questions about development. For example, it is unknown whether attenuated symptoms represent similar risk in young adolescents versus adults. For example, it has been suggested that some symptoms may be more transient in early adolescence, whereas they may represent more stable risk markers when observed in adults ${ }^{9}$. Despite challenges associated with substantial variability among findings to date, extant studies converge in highlighting the utility of UHR criteria for detecting individuals at significantly heightened risk of developing a psychotic disorder, relative to the general population. For example, in the largest UHR studies to date, relative risk was found to be as high as $405^{2}$ and $1630^{8}$. 
Table 1. Summary of rates of transition to psychosis

\begin{tabular}{|l|c|c|c|}
\hline Study & $\mathbf{N}$ & Rate & Follow-up \\
\hline Bruderholz Study (Simon \& Umbricht, 2010) & 42 & $17 \%$ & 12 months \\
\hline CARE (Haroun et al., 2006) & 50 & $15 \%$ & 12 months \\
\hline EDIE (Morrison et al., 2004) & 23 & $22 \%$ & 12 months \\
\hline EPOS (Ruhrmann et al., 2010) & 245 & $19 \%$ & 29 months \\
\hline NAPLS (Cannon et al., 2008) & 291 & $35 \%$ & 12 months \\
\hline PACE (Yung et al., 2004) & & & 12 months \\
\hline PRIME (Miller et al., 2002) & 13 & $54 \%$ & 6 months \\
\hline RAP (Cornblatt et al., 2003) & 34 & $27 \%$ & \\
\hline
\end{tabular}

Enhancing prediction: multivariate prediction models

The early detection and accurate identification of individuals at increased risk represents a critical step for early intervention and the potential to delay or even prevent psychosis. As evidenced by reported transition rates, current criteria for at-risk mental states have strong predictive validity; however, high rates of non-conversion, representing potential "false positive" cases, underline the need for improved prediction algorithms. Enhancing prediction allows for improved accuracy of identification and can better inform the timing and need for interventions. In addition, increasing diagnostic accuracy serves to reduce stigma and exposure to potential adverse events among individuals who do not transition to psychosis. As such, research has increasingly shifted toward efforts to improve upon extant prediction models, particularly using multivariate algorithms that seek to optimize prediction. These studies have also modeled the survival curve associated with transition to psychosis, allowing for increased understanding of changes in the rate of transition across time and periods of relatively higher risk.

As part of the Personal Assessment and Crisis Evaluation (PACE) study, Yung and colleagues examined the predictive power of clinical variables assessed at baseline and their combination to improve prediction beyond UHR criteria ${ }^{1}$. Over a follow-up period of 12 months, 20 of 49 individuals developed psychosis, yielding a transition rate of $40.8 \%$. A long duration of prodromal symptoms, poor functioning at intake, low-grade psychotic symptoms, and depression and disorganization were identified as highly significant predictors of transition. Following the identification of significant predictors in univariate analyses, Yung et al. examined the risk of conversion given two or more, three or more, four or more, and five or more of the potential predictors ${ }^{1}$. Having four or more of the potential predictors yielded the strongest prediction, leading to a "four-or-more" strategy of risk identification. When each participant was classified as positive or negative according to this strategy, results demonstrated that the majority of participants with four or more of the predictors at baseline transitioned to psychosis within six months. This prediction algorithm was associated with a positive predictive value of $80 \%$ and a hazard ratio of approximately 30 . It also had good specificity (91\%), sensitivity $(86 \%)$, and negative predictive value $(94 \%)$. In addition, Yung and colleagues examined the survival curve, revealing that the greatest risk of transition to psychosis occurred within 4.5 months of ascertainment ${ }^{1}$. Specifically, $70 \%$ of all transitions happened within 4.5 months in this study sample. As such, these findings improved prediction over and above UHR criteria alone and elucidated changes in the transition rate over time.

In the largest UHR study to date, the North American Prodrome Longitudinal Study (NAPLS) consortium empirically derived multivariate algorithms to enhance prediction and examined the survival curve associated with transition to psychosis among 291 individuals meeting UHR criteria ${ }^{2}$. Participants met APS, BIPS, or GRD criteria at study entry and were re-assessed using the SIPS every six months for a follow-up duration of 2.5 years. Participants 
were recruited from eight clinical research centers, with the large sample size allowing for enhanced statistical reliability. Examination of the survival curve revealed a decelerating trend, such that increasingly fewer individuals transitioned to psychosis as time passed. The incidence rates of transition were $13 \%, 9 \%$, $5 \%$, and $2.7 \%$ for periods of the first 6 months, $7-12$ months, each 6-month epoch from 3-24 months, and 25-30 months, respectively. The cumulative prevalence rates of transition were $12.7 \%, 21.7 \%, 26.8 \%, 32.6 \%$, and $35.3 \%$ at $6,12,18,24$, and 30 months, respectively. Thus, the UHR criteria alone were associated with positive predictive power of $35 \%$ over the 2.5 -year follow-up duration. Of note, no participants in a control group $(n=134)$ transitioned during that period. Consistent with the survival function observed in the PACE study, these results suggested that the UHR criteria are sensitive to individuals at imminent risk for the onset of psychosis. In addition, they provided information that is critical to informing the timing of interventions.

With regard to multivariate prediction of psychosis, results from the NAPLS study demonstrated substantial increases in positive predictive power using empirically derived combinations of predictor variables. Over 70 potential predictor variables measured at baseline assessment were screened across a variety of domains and tested to eliminate redundancy, resulting in five predictors that related uniquely and significantly to transition to psychosis. These significant predictors were genetic risk for psychosis with recent functional deterioration, higher levels of unusual thought content, higher levels of suspicion/paranoia, more severe social impairment, and a history of drug abuse. At the univariate level, each of the significant predictors had superior positive predictive power (43-52\%) to the UHR criteria alone, and the majority had good sensitivity. Combining these risk factors into multivariate algorithms led to additional increases in positive predictive power. Among two-predictor models, the combination of GRD and unusual thought content, or GRD and impaired social functioning, had the highest positive predictive power $(69 \%$ and $61 \%$, respectively), though sensitivity was reduced (38\% and $55 \%$, respectively). Certain three-factor models provided additional improvement in positive predictive power, though with a tradeoff in reduced sensitivity. Specifically, the combination of GRD, unusual thought content, and either suspicion/paranoia or social impairment was associated with positive predictive powers of $74 \%$ and $81 \%$, respectively, with only slightly lower sensitivity $(34 \%$ and $30 \%$, respectively). Four- and five-factor models were not associated with incremental increases in positive predictive power.

Of note, treatment with antipsychotic medication during the follow-up period was related to transition to psychosis at the univariate level; however, the association was no longer significant in the multivariate analysis. In addition, controlling for antipsychotic use did not significantly affect the results of the univariate or multivariate prediction algorithms. These findings are likely due to the tendency for prescription of antipsychotic medication among individuals with greater symptom severity. In this study, multivariate prediction algorithms provided enhanced positive predictive power but at the expense of sensitivity, which may result from the lower base rate of co-occurring risk factors. Allowing for non-co-occurring combinations of risk factors yielded increased sensitivity but reduced positive predictive power, highlighting the trade-offs inherent to these prediction algorithms. Overall, the NAPLS study demonstrated impressive positive predictive power using empirical derivation of multivariate algorithms, and it is likely that sensitivity may be increased through the incorporation of measures such as brain-based or neurocognitive indices in the future.

Building on previous research aimed at improving the prediction of psychosis, the European Prediction of Psychosis Study (EPOS) sought to further develop high-risk criteria to be applied at the level of individual risk assessment ${ }^{8}$. The EPOS study consisted of a prospective multisite naturalistic study with a sample of 245 participants and follow-up assessments conducted at 9 and 18 months. A strength of this study was its concurrent examination of UHR and basic symptom criteria, such that participants met UHR criteria and/or basic symptom-based criteria, as defined by cognitive disturbances (COGDIS) on the BSABS. The instantaneous incidence rates of transition were $7 \%, 11 \%, 14 \%$, and $19 \%$ after $6,9,12$, and 18 months, respectively. Rates did not significantly differ for participants who were positive for UHR only, versus COGDIS only, versus positive for both UHR and COGDIS. However, the co-occurrence of UHR and COGDIS symptoms yielded a higher sensitivity. In order to calculate a prognostic index (PI) for each individual participant, Ruhrmann and colleagues first empirically derived six variables that were highly predictive of transition to psychosis ${ }^{8}$. The six predictor variables were SIPS positive subscale score $>16$, SIPS bizarre thinking score $>2$, SIPS sleep disturbances score $>2$, schizotypal personality disorder according to the SIPS, GAF score (highest in past year), and years of education (including university). The final Cox regression model was then applied to each participant, with the mean and positive and negative standard deviations used to stratify the sample into four classifications of risk.

Ruhrmann and colleagues proposed a two-step procedure, in which help-seeking individuals are first screened using UHR and COGDIS criteria and are then classified using the PI prediction model $^{8}$. An advantage to this model is increased sensitivity to individuals who might benefit from early intervention, as it continues to consider a participant who has met inclusion criteria to be at-risk, regardless of his PI score. In addition, such a risk staging model allows for interventions that are targeted and adjusted based on PI classification. For example, it might be that psychotherapy and monitoring are appropriate for a lower risk class, whereas low-dose antipsychotic treatment may be warranted for a higher class of risk.

Several predictors of conversion to psychosis replicated across the PACE, NAPLS, and EPOS studies ${ }^{1,2,8}$. As would be expected, higher levels of subthreshold positive symptoms (i.e., APS) were consistently observed to predict psychosis. Specifically, overall lowgrade psychotic symptoms were predictive in the PACE and EPOS 
studies, and more specific symptoms were identified in the NAPLS (unusual thought content, suspiciousness/paranoia) and EPOS (bizarre thinking) studies. Poor functioning, especially a decrease from typical functioning, was also associated with conversion in all three studies. Social functioning may be particularly predictive of conversion. For example, it contributed to overall GAF scores as measured in the PACE and EPOS studies, and it was specifically found to be predictive of conversion in the NAPLS study. Finally, though specific findings varied, some form of genetic risk (sometimes in combination with functional deterioration) was predictive of conversion across these studies. For example, GRD in combination with APS was associated with increased predictive power and sensitivity in the PACE study ${ }^{13}$. GRD also predicted conversion in the NAPLS study, to a greater extent than family history or schizotypal personality disorder alone. In the EPOS study, schizotypal personality disorder was predictive of conversion, though family history (even in combination with functional deterioration) was not. Based on these findings, severity of subthreshold positive symptoms, poorer functioning, and genetic risk appear to be consistent predictors of conversion to psychosis. Taken together, these predictors suggest that the transition to psychosis is marked by inherited vulnerability in the context of changes in thinking and functioning.

\section{Outcomes of non-converters}

Despite improvements in the prediction of transition to psychosis, studies have consistently observed that the majority of individuals ascertained according to UHR criteria do not develop psychosis. Given potential stigma, exposure to adverse events, and limited resources for early intervention, it is critical to understand whether these individuals truly represent "false positive" cases and whether they would benefit from monitoring or early intervention. Recently, research has increasingly focused on characterizing non-converting individuals and examining the trajectories of their symptoms and functioning.

In the Bruderholz Study, Simon and Umbricht reported outcomes after one year of follow-up for participants who initially met UHR criteria ${ }^{11}$. In the sample of 52 participants, $13.5 \%$ transitioned to psychosis, $26.5 \%$ had sustained UHR features, and $59.2 \%$ fully remitted after one year. As such, the likelihood of remission was over four times greater than the likelihood of transition. Non-cases (i.e., non-converters) did not differ from cases (i.e., converters and participants with sustained UHR features) on socio-demographic or clinical variables at baseline. In addition, medication and treatment status were not related to outcome after one year. Similarly, Ziermans and colleagues found that $49.1 \%$ of individuals at UHR for psychosis $(n=57)$ had remitted from UHR status after a two-year follow-up period, whereas $15.6 \%$ had transitioned to psychosis and $35.3 \%$ continued to meet UHR criteria ${ }^{12}$. No differences on socio-demographic or clinical variables at baseline were observed between the groups with differing outcomes. These results highlight the heterogeneity among at-risk samples and the need for continued refinement of
UHR criteria; however, future research with longer follow-up periods will be critical to understanding what happens over time among individuals who do not transition to psychosis.

In order to better characterize non-converting individuals, Addington and colleagues (in press) examined clinical and functional outcomes among non-converters in the NAPLS study who had follow-up data for at least 1-2 years $(\mathrm{n}=111)$ and also compared them with a matched sample of non-psychiatric control participants $(\mathrm{n}=111)^{14}$. Results demonstrated that the UHR participants who did not transition to psychosis within the study period demonstrated improved symptoms and functioning over time. Specifically, their positive and negative symptoms both improved between baseline and one-year follow-up. Social and role functioning improved from baseline to one-year follow-up, and global functioning improved from baseline to one-year and from one-year to two-year follow-up. Nevertheless, despite these improvements, social and role functioning remained poorer among non-converters than among control participants. In addition, only $42.9 \%$ of non-converters had at least one attenuated positive symptom at one-year follow-up ( $40.8 \%$ at two-year follow-up). Because APS criteria require the onset or worsening of symptoms within the past 12 months, only $5.4 \%$ of non-converters met APS criteria again after one or two years, even though all non-converters met APS criteria at baseline. Comparisons of participants with sustained versus remitted attenuated positive symptoms did not reveal differences in age, gender, or functioning at baseline. Finally, though a substantial number of non-converters had a diagnosis of a mood or anxiety disorder at follow-up, the number of diagnoses decreased over time. Despite improvement in a subgroup of non-converters, findings from this study suggest that UHR criteria are associated with lasting disability. In addition, they demonstrate that initial APS criteria do not relate to the persistence of severe symptoms in all cases. Thus, future research may improve prediction by expanding beyond clinical predictors of psychosis and by further considering how to best address the issue of non-converters (e.g., improved symptoms but persistent functional disability).

Recently, Schlosser and colleagues (in press) examined factors that predict recovery and trajectories of symptoms and functioning among non-converters in a UHR sample from the Center for the Assessment and Prevention of Prodromal States at UCLA $^{15}$. Across the two-year longitudinal study, $32 \%$ of the sample transitioned to psychosis. Competing risk survival analyses were used to estimate a $30 \%$ rate of conversion, $36 \%$ rate of symptomatic remission, and $30 \%$ rate of functional recovery by two years. Interestingly, of 57 participants who did not transition to psychosis, 17 experienced both symptomatic remission and functional recovery, 15 experienced symptomatic remission but not functional recovery, and 15 experienced functional recovery but not symptomatic remission. Using proportional causespecific hazards models, Schlosser et al. (in press) tested whether time-varying symptoms and functioning affected conversion, symptom remission, and functional recovery ${ }^{15}$. Negative 
symptoms strongly predicted conversion, with a $10 \%$ increase in hazard of conversion associated with each one-point increase in negative symptoms. Greater social functioning was associated with a reduced risk of conversion. In addition, greater symptom severity related to lower remission rates, and higher functioning related to higher remission rates. Specifically, negative symptoms, dysphoric mood/anxiety symptoms, social functioning, and role functioning all significantly predicted remission rates. However, in a combined model, only mood/anxiety symptoms remained significant, suggesting redundancy between the variables. Finally, negative symptoms were also associated with functional recovery. In addition to examining time-varying models, Schlosser et al. (in press) examined whether baseline measures of symptoms and functioning predicted outcomes ${ }^{15}$. Negative symptoms at baseline significantly predicted conversion when symptomatic remission, but not functional recovery, was the competing event. Baseline negative symptoms were also associated with subsequent functional recovery. Finally, baseline social functioning significantly predicted conversion when symptomatic remission was the competing event. These findings highlight the importance and predictive power of negative symptoms and reveal predictors of more favorable outcomes.

In order to examine the course of change, trajectory plots demonstrated how positive symptoms, negative symptoms, mood/ anxiety symptoms, and social and role functioning changed over time depending on outcome. Results demonstrated that these trajectories clustered by outcome group, such that individuals who remitted with the highest functioning resembled levels of healthy controls, whereas trajectories of individuals who neither converted nor remitted fell between the levels of converters and participants who remitted. Interestingly, the plots also revealed that psychosocial functioning was more temporally stable than symptoms for the majority of UHR participants. Overall, nonconverters whose symptoms remitted exhibited normative courses of both symptoms and functioning, and they initially presented with lower severity of symptoms. Thus, future efforts to refine CHR criteria may benefit from eliminating cases who present with low-grade APS or who show early improvements in order to decrease the identification of false positives.

\section{Conclusions and future directions}

Research on the prediction of psychosis onset has provided substantial evidence of the ability to prospectively identify individuals at increased imminent risk for psychosis relative to the general population. In particular, novel methods for improving prediction, such as multivariate algorithms and risk classification models, have enhanced accuracy and predictive power. Achieving higher sensitivity and the cross-validation of these algorithms on independent samples remain tasks for future research. Moreover, the continued refinement of criteria for predicting psychosis will likely benefit from the integration of more quantitative, objective markers such as brain-based measures and neurocognitive performance. However, despite significant improvements in the prediction of psychosis onset, the majority of individuals identified as UHR do not convert to psychosis. Recent studies suggest that these individuals show more favorable outcomes in terms of symptoms and functioning, but that many still remain impaired relative to healthy controls. In general it appears that about $1 / 3$ of UHR cases convert to psychosis, about $1 / 3$ do not convert but remain symptomatic and functionally impaired, and about $1 / 3$ recover symptomatically and functionally. Thus, an important aspect of future research will be addressing the issue of potential false positive cases and improving understanding of their needs (e.g., appropriate period of monitoring, how to best direct limited resources). Continued efforts to detect early risk for psychosis are critical for informing early intervention and provide increasing promise of delaying or even preventing the onset of psychosis. 


\section{Disclosures and Role of Funding Sources}

\begin{tabular}{|c|c|c|c|c|c|c|c|}
\hline $\begin{array}{l}\text { Writing group } \\
\text { member }\end{array}$ & Employment & $\begin{array}{c}\text { Research } \\
\text { grant }\end{array}$ & $\begin{array}{c}\text { Other research } \\
\text { grant or } \\
\text { medical } \\
\text { continuous } \\
\text { education }{ }^{2}\end{array}$ & $\begin{array}{l}\text { Speaker's } \\
\text { honoraria }\end{array}$ & $\begin{array}{l}\text { Ownership } \\
\text { interest }\end{array}$ & $\begin{array}{c}\text { Consultant/ } \\
\text { Advisory board }\end{array}$ & Other $^{3}$ \\
\hline Dylan G. Gee & -- & $\begin{array}{l}\text { National } \\
\text { Science } \\
\text { Foundation } \\
\text { Graduate } \\
\text { Research } \\
\text { Fellowship }\end{array}$ & --- & -- & --- & -- & --- \\
\hline Tyrone D. Cannon & --- & $\begin{array}{c}\text { National } \\
\text { Institute of } \\
\text { Mental Health } \\
\text { grant } \\
\text { MH081902 }\end{array}$ & --- & --- & --- & -- & $\begin{array}{l}\text { Gifts to the } \\
\text { UCLA } \\
\text { Foundation } \\
\text { from Garen } \\
\text { and Shari } \\
\text { Staglin and the } \\
\text { International } \\
\text { Mental Health } \\
\text { Research } \\
\text { Organization } \\
\text { (IMHRO) }\end{array}$ \\
\hline
\end{tabular}

* Modest

** Significant

*** Significant: Amounts given to the author's institution or to a colleague for research in which the author has participation, not directly to the author.

\section{References}

1. Yung AR, Phillips LJ, Pan Yuen H, Francey SM, McFarlane CA, Hallgren M, McGorry PD. Psychosis prediction: 12-month follow up of a high-risk ("prodromal") group. Schizophr Res.2003;60(1):21-32.

2. Cannon TD, Cadenhead K, Cornblatt B, Woods SW, Addington J, Walker E, Seidman LJ, Perkins D, Tsuang M, McGlashan T, Heinssen R. Prediction of psychosis in youth at clinical high risk: a multisite longitudinal study in North America. Arch Gen Psychiatry. 2008;65(1):28-37.

3. Yung A, Phillips L, McGorry P, Ward J, Donovan K, Thompson K. Comprehensive assessment of at risk mental states (CAARMS). Melbourne: PACE Clinic, University of Melbourne, Department of Psychiatry; 2002

4. McGlashan TH, Miller TJ, Woods SW, Rosen JL, Hoffman RE, Davidson L. Structured clinical interview for prodromal syndromes. Yale School of Medicine: PRIME Research Clinic; 2001.

5. Gross $\mathrm{G}$, Huber $\mathrm{G}$, Klosterkotter J. Bonn scale for the assessment of basic symptoms - BSABS. Berlin: Springer; 1987.

6. Schultze-Lutter F, Wieneke A, Picker $\mathrm{H}$. The schizophrenia prediction instrument -adult version (SPI-A). Schizophr Res. 2004;70:76-77.

7. Klosterkotter J, Hellmich M, Steinmeyer EM, Schultze-Lutter F. Diagnosing schizophrenia in the initial prodromal phase. Arch Gen Psychiatry. 2001;58(1):158-64.

8. Ruhrmann S, Schultze-Lutter F, Salokangas RK, Heinimaa M, Linszen D, Dingemans P, Birchwood M, Patterson P, Juckel G, Heinz A, Morrison A, Lewis S, von Reventlow HG, Klosterkötter J. Prediction of psychosis in adolescents and young adults at high risk. Arch Gen Psychiatry. 2010;67(3):241-51.

9. Olsen KA, Rosenbaum B. Prospective investigations of the prodromal state of schizophrenia: assessment instruments. Acta Psychiatr Scand. 2006;113(4):247-72.

10. Yung AR, Nelson B, Stanford C, Simmons MB, Cosgrave EM, Killackey E, Phillips LJ, Bechdolf A, Buckby J, McGorry PD. Validation of "prodromal" criteria to detect individuals at ultra high risk of psychosis: 2 year follow-up. Schizophr Res. 2008;105(1-3):10-17.

11. Simon AE, Umbricht D. High remission rates from an initial ultra-high risk state for psychosis. Schizophr Res. 2010; 116(2-3):168-72.

12. Ziermans TB, Schothorst PF, Sprong M, van Engeland H. Transition and remission in adolescents at ultra-high risk for psychosis. Schizophr Res. 2011;126(13):58-64.
13. Yung AR, Phillips LJ, Yuen HP, McGorry PD. Risk factors for psychosis in an ultra high-risk group: psychopathology and clinical features. Schizophr Res. 2004;67(2-3):131-42.

14. Addington J, Cornblatt BA, Cadenhead KS, Cannon TD, McGlashan TH, Perkins DO, Seidman LJ, Tsuang MT, Walker EF, Woods SW, Heinssen R. At clinical high risk for psychosis: Outcome for nonconverters. Am J Psychiatry. 2011;168(8):800-5.

15. Schlosser DA, Jacobson S, Chen Q, Sugar CA, Niendam TA, Li G, Bearden $\mathrm{CE}$, Cannon TD. Recovery from an at-risk state: Clinical and functional outcomes of putatively prodromal youth who do not develop psychosis. Schizophr Bull..In press 2011.

16. Haroun N, Dunn L, Harouna A, Cadenhead KS. Risk and protection in prodromal schizophrenia: ethical implications for clinical practice and future research. Schizophr Bull. 2006;32(1):166-78.

17. Morrison AP, French P, Walford L, Lewis SW, Kilcommons A, Green J, Parker S, Bentall RP.. Cognitive therapy for the prevention of psychosis in people at ultra-high risk. Randomized controlled trial. Br J Psychiatry. 2004;185: 291-97.

18. Miller TJ, McGlashan TH, Rosen JL, Somjee L, Markovich PJ, Stein K, Woods SW.. Prospective diagnosis of the initial prodrome for schizophrenia based on the Structure Interview for Prodromal Syndromes: Preliminary evidence of interrater reliability and predictive validity. Am J Psychiatry. 2002;159(5):863-65.

19. Cornblatt BA, Lencz T, Smith CW, Correll CU, Auther AM,, Nakayama E. The schizophrenia prodrome revisited: a neurodevelopmental perspective. Schizophr Bull. 2003;29(4):633-51. 